\title{
Suplementary Information
}

\section{Probing the Fate of Mn Complexes in Nafion: A Combined Multi-Frequency EPR and XAS Study}

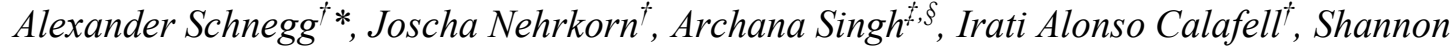 \\ Bonke $^{*}$, Rosalie Hocking ${ }^{\#}$, Klaus Lips ${ }^{\dagger}$ and Leone Spiccia ${ }^{\dagger}$
}

$\dagger$ Berlin Joint EPR Laboratory (BeJEL), Institute Nanospectroscopy, Helmholtz-Zentrum Berlin für Materialien und Energie, Kekuléstr. 5, 12489, Berlin, Germany

$\$$ School of Chemistry and Australian Centre of Excellence for Electromaterials Science, Monash University, Victoria, 3800, Australia.

$\S$ INSPIRE Faculty, Council of Science and Industrial Research Center-AMPRI, Hoshangabad Road, Bhopal, MP 462026

\# Discipline of Chemistry, School of Pharmacy and Molecular Sciences, James Cook University, Townsville, 4811

*alexander.schnegg@helmholtz-berlin.de 

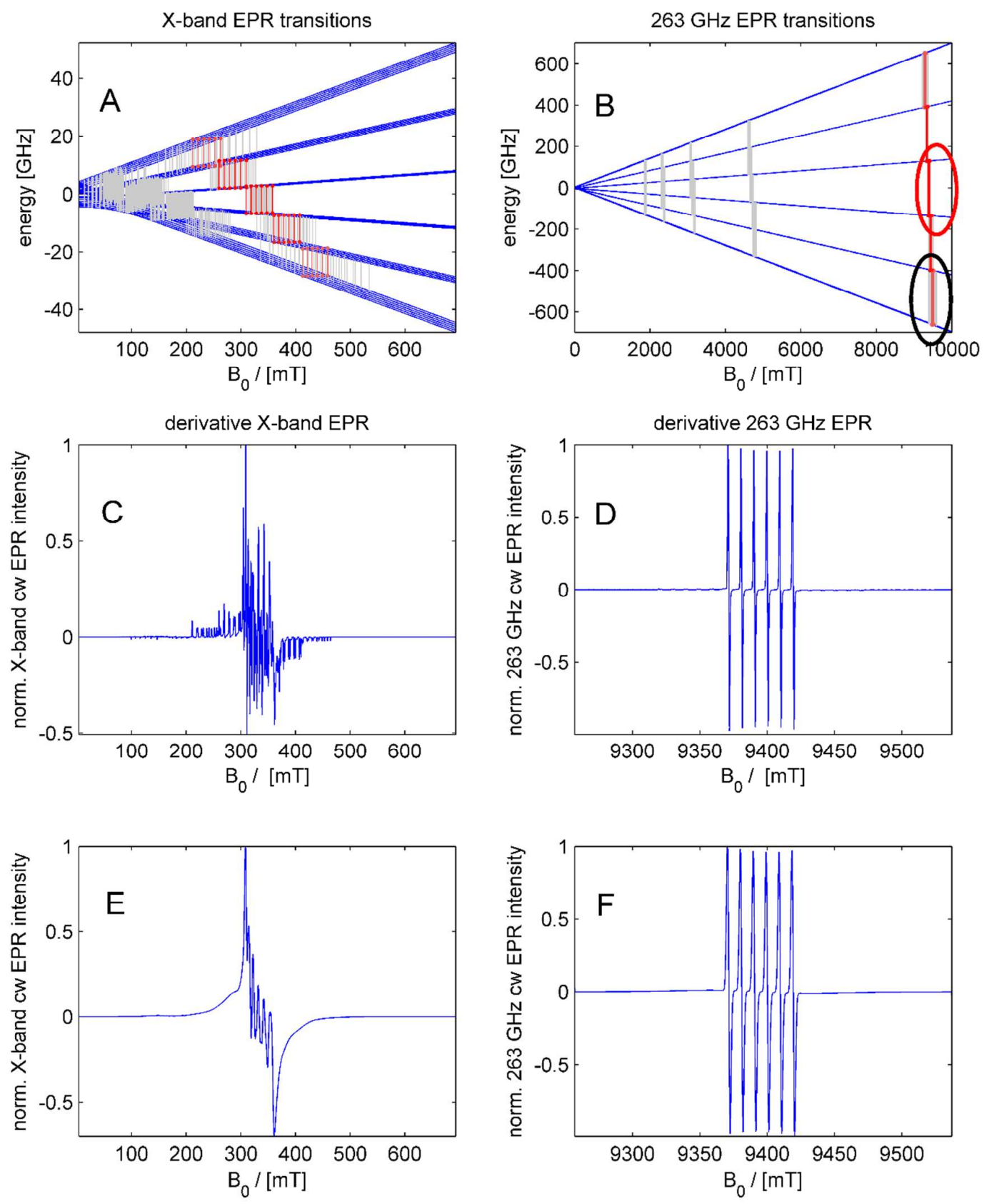

SI Fig. 1: Top: Mn(II) spin energy levels as a function of the external magnetic field together with EPR transitions for microwave excitation energies of $9.3 \mathrm{GHz}$ (A) and $263 \mathrm{GHz}$ (B) (blue: $\mathrm{m}_{\mathrm{s}}$ sublevels, grey: partly forbidden transitions, red: allowed transitions). Circles in the high field energy level diagram (B) indicate the dominating transitions at high (red) and low (black) temperatures, respectively. In plots (C) to (F), X band and $263 \mathrm{GHz}$ derivative EPR spectra calculated from these transitions are depicted. Spin energy levels and EPR spectra were calculated with Easyspin a Matlab toolbox [1] and with the following set of spin Hamiltonian parameters: $A_{\text {iso }}=268 \mathrm{MHz}, g=2.00071, D=700 \mathrm{MHz}, E=250 \mathrm{MHz}$. In spectra (C) and (D) line broadening due to a distribution of $D$ and $E$ was neglected, while spectra (E) and (F) were calculated assuming a Gaussian distribution of $D$ and $E$ values with full width at half height $D$ Strain $=700 \mathrm{MHz}$ and $E$ Strain $=200 \mathrm{MHz}$, respectively. 

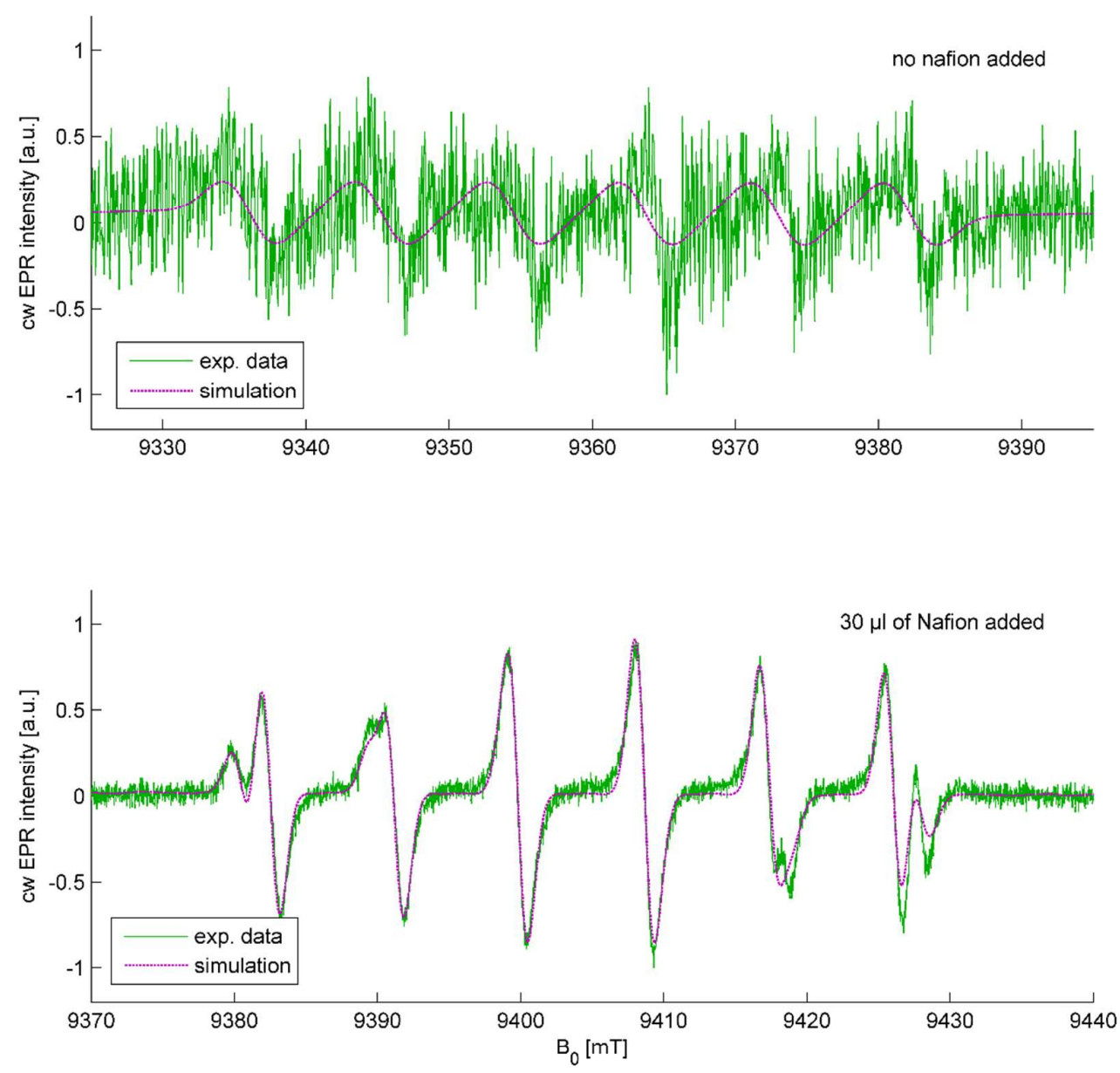

SI Fig. 2: Top: $263 \mathrm{GHz}$ cw EPR spectrum of $30 \mu \mathrm{l}$ of frozen $8 \mathrm{mM} \mathrm{Mn}{ }^{\text {IV }}$ acetonitrile solution. Bottom: $263 \mathrm{GHz} \mathrm{cw}$ EPR spectrum of the same solution after adding $30 \mu 1$ of dried Nafion film. Green experimental spectra measured at $T=150 \mathrm{~K}$ and $v_{\mathrm{mw}}=262.07 \mathrm{GHz}$ (top) and $v_{\mathrm{mw}}=263.33 \mathrm{GHz}$ (bottom), respectively. Blue: Simulations assuming one (top) and two (bottom ) $\mathrm{Mn}$ (II) states with different spin coupling parameters, respectively. Top: $\mathrm{Mn}(\mathrm{II}), g_{\text {iso }}$ $=2.0007, A_{\text {iso }}=258 \mathrm{MHz}, D=800 \mathrm{MHz}, E=150 \mathrm{MHz}$; Bottom: Mn(II) species A, $g_{\text {iso }}=$ $2.0006, A_{\text {iso }}=265 \mathrm{MHz}, D=780 \mathrm{MHz}, E=150 \mathrm{MHz}$, weight $0.37 ; \mathrm{Mn}$ (II) species $\mathrm{B}, g_{\text {iso }}=$ $2.0006, A_{\text {iso }}=244 \mathrm{MHz}, D=780 \mathrm{MHz}, E=150 \mathrm{MHz}$, weight 0.78 . 

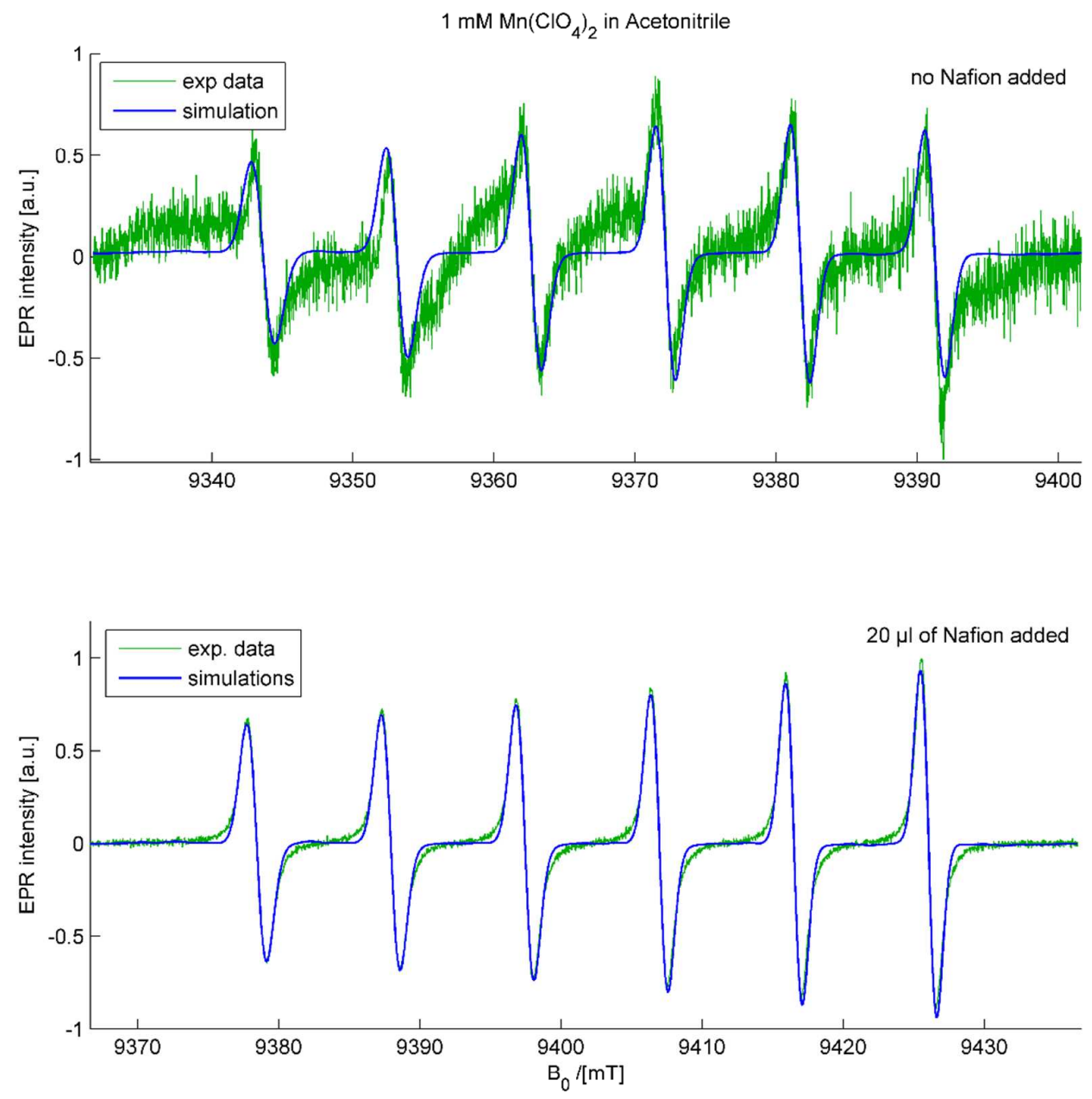

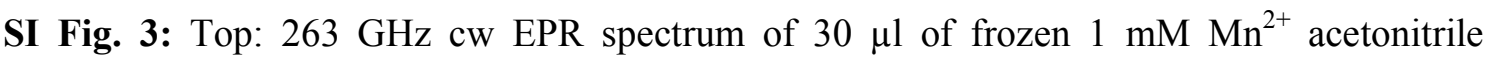
solution. Bottom: $263 \mathrm{GHz}$ cw EPR spectrum of the same solution after adding $20 \mu \mathrm{l}$ of dried Nafion film. Green experimental spectra measured at $T=150 \mathrm{~K}$ and $v_{\mathrm{mw}}=262.328 \mathrm{GHz}$ (top) and $v_{\mathrm{mw}}=263.276 \mathrm{GHz}$ (bottom), respectively. Blue: Simulations assuming $\mathrm{Mn}(\mathrm{II})$ states with different spin coupling parameters: Top: $\mathrm{Mn}(\mathrm{II}), g_{\text {iso }}=2.0008, A_{\text {iso }}=267 \mathrm{MHz}, D=800$ $\mathrm{MHz}, E=150 \mathrm{MHz}$; Bottom: $\mathrm{Mn}(\mathrm{II}), g_{\text {iso }}=2.0006, A_{\text {iso }}=267 \mathrm{MHz}, D=780 \mathrm{MHz}, E=150$ $\mathrm{MHz}$. 

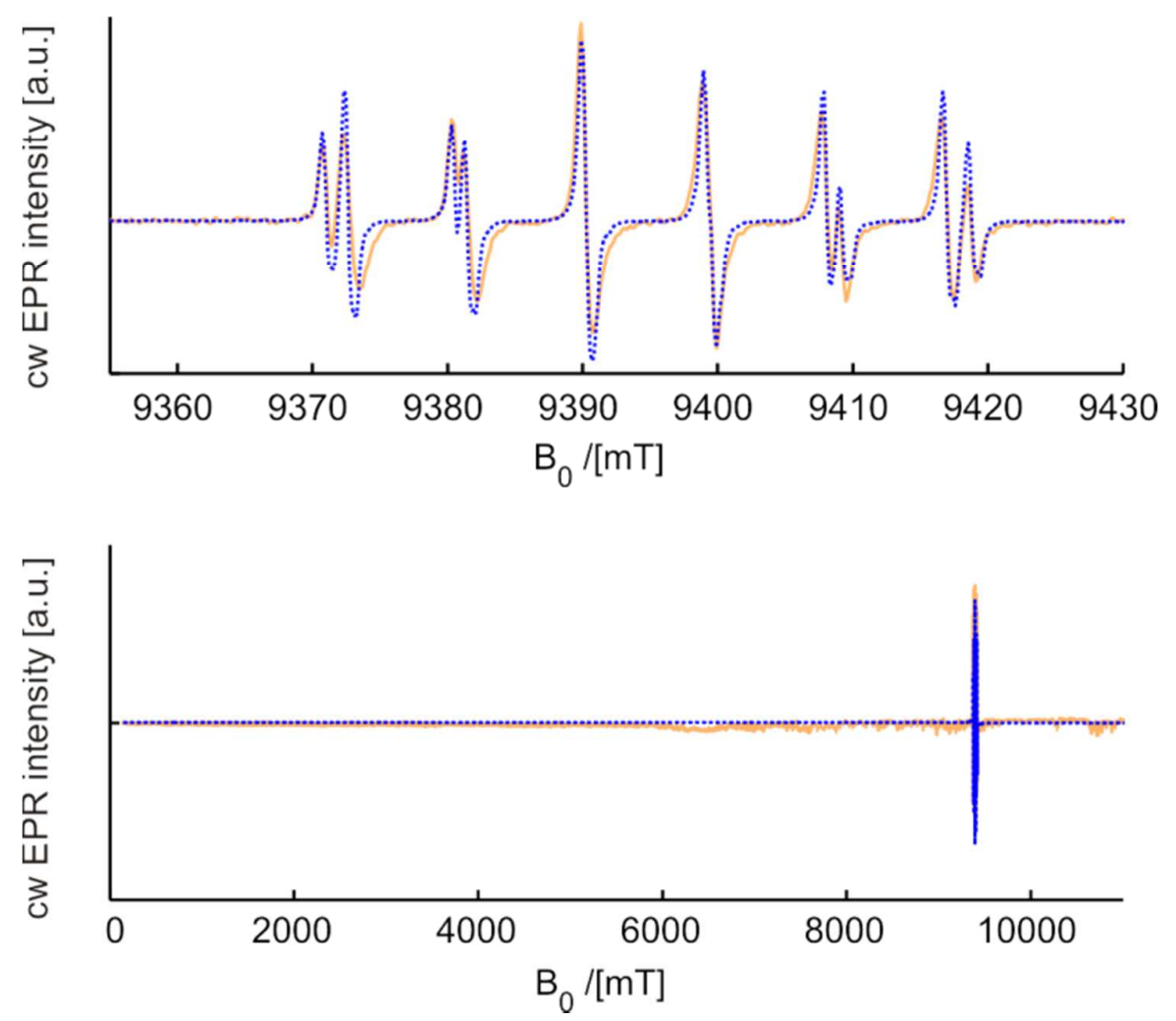

SI Fig. 4: $263 \mathrm{GHz}$ EPR spectra (orange) of the electro oxidized product of $\mathrm{Mn}^{\mathrm{IV}}$ doped in Nafion films (State 2) with a magnetic field sweep width of $75 \mathrm{mT}$ (top) and $11 \mathrm{~T}$ (bottom). Green: experimental spectra measured at $T=25 \mathrm{~K}$ and $v_{\mathrm{mw}}=263.09 \mathrm{GHz}$. Blue: spectral simulations assuming a superposition of two Mn(II) species A ( $g_{\text {iso }}=2.0008(1), A_{\text {iso }}=269(1)$, $D=860(200) \mathrm{MHz}$ and $E=215(100) \mathrm{MHz}$ for $\left.\mathrm{Mn}^{\mathrm{IV}}\right)$ and $\mathrm{B}\left(g_{\text {iso }}=2.0008\right.$ and $A_{\text {iso }}=248$ $\mathrm{MHz}, D=780 \mathrm{MHz}, E=150 \mathrm{MHz}$,). Note, the resonance at $9400 \mathrm{mT}$ in the lower panel is the same signal as in the upper panel. However, due to the finite line width of the plot the 10 individual resonance lines cannot be resolved. 


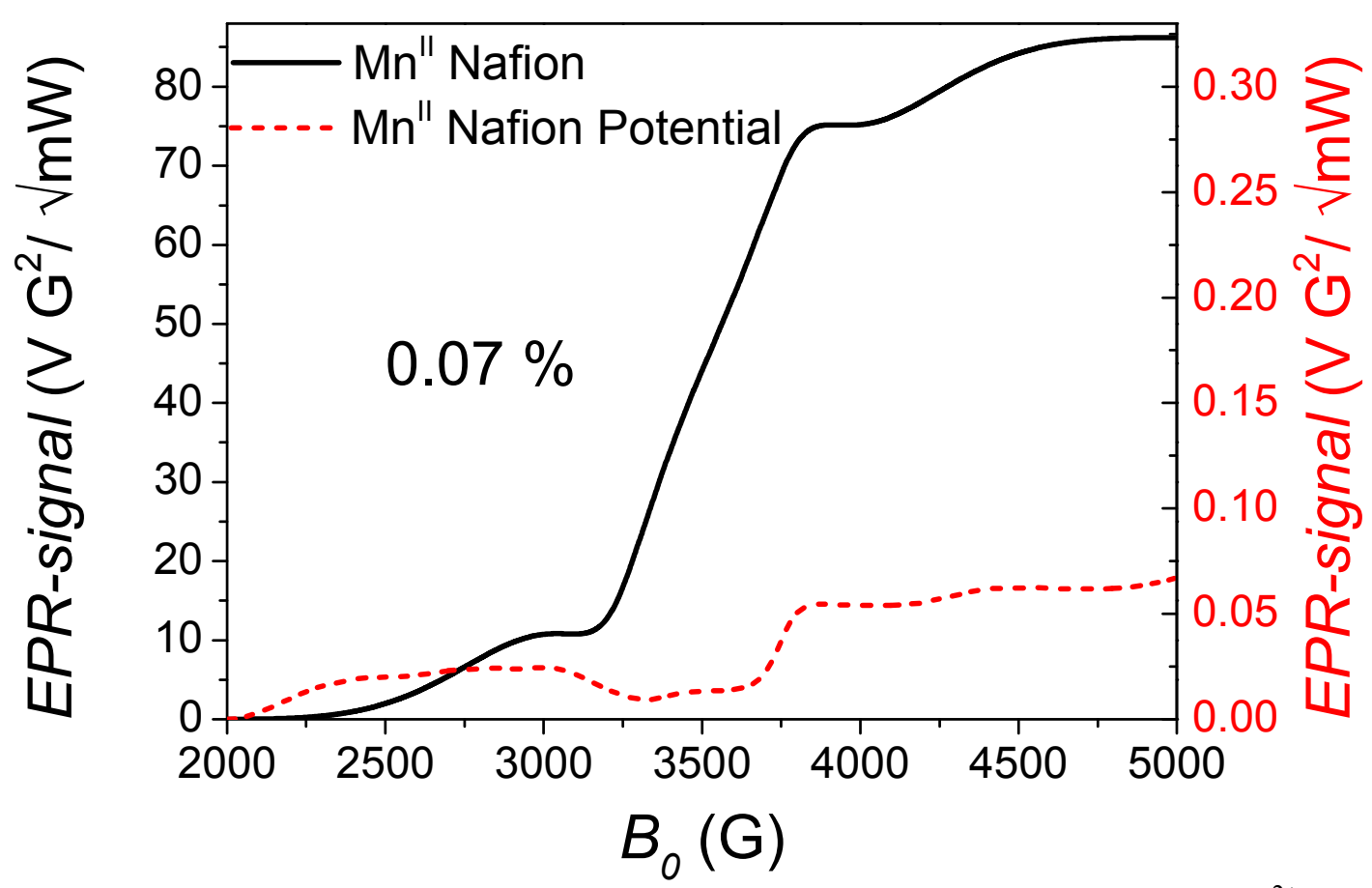

SI Fig. 5: Double Integrated X-band EPR signal of Nafion films doped with $\mathrm{Mn}^{2+}$ before (solid black) and after electro oxidation (dotted red line). The data is normalized to the microwave power, as the films were measured with different attenuations. $0.07 \%$ indicates the remaining $\mathrm{Mn}(\mathrm{II})$ content after applying a potential bias of $1.1 \mathrm{~V}$.

1. Stoll, S. and A. Schweiger, EasySpin, a comprehensive software package for spectral simulation and analysis in EPR. Journal of Magnetic Resonance, 2006. 178(1): p. 42-55. 